\title{
Optimization Method for Solution Model of Laser Tracker Multilateration Measurement
}

\author{
Hongfang Chen, Zhi Tan, Zhaoyao Shi, Huixu Song and Hao Yan \\ Beijing Engineering Research Center of Precision Measurement Technology and Instruments, College of Mechanical \\ Engineering and Applied Electronics Technology, Beijing University of Technology, Beijing 100000, China
}

\begin{abstract}
Multilateration measurement using laser trackers suffers from a cumbersome solution method for high-precision measurements. Errors are induced by the self-calibration routines of the laser tracker software. This paper describes an optimization solution model for laser tracker multilateration measurement, which effectively inhibits the negative effect of this self-calibration, and further, analyzes the accuracy of the singular value decomposition for the described solution model. Experimental verification for the solution model based on laser tracker and coordinate measuring machine (CMM) was performed. The experiment results show that the described optimization model for laser tracker multilateration measurement has good accuracy control, and has potentially broad application in the field of laser tracker spatial localization.
\end{abstract}

Keywords: Multilateration measurement, solution model, singular value decomposition, laser tracker.

\section{INTRODUCTION}

The emergence of laser tracker had a huge impact on three-dimensional space measurement [1]. Since then laser trackers have been widely used in industries for large-scale metrology in a range of applications including the assembly of large structures, reverse engineering and the volumetric accuracy checking of large CMMs and machine tools, and they have played a critical role in aerospace and defense industries [2].

A laser tracker is a portable coordinate measuring system, used for measuring the azimuth and distance through a lens to obtain 3D coordinates of the target mirror, in cooperation with the objective lens of the target. The measurement information, which LaserTRACER gets, does not contain angular information. So in the multilateration measurement of LaserTRACER it can be compiled to reduce the uncertainty.

At present, multilateration measurement has been widely developed [3]-[5]. Zhang et al. from the engineering college of Durham University made further study of optimization of multilateration set-ups and measurement plans [6]. Wang Jindong applied the multi-station and time-sharing measurement to machine tool correction [7]-[9].

The process of calculating multilateration measurement model is very tedious in practice. Self-calibration errors in laser tracker stations are introduced by the usual solution model. This article describes a new solution model of laser tracker multilateration measurement, researches the accuracy of singular value decomposition for the described solution model. Experiments are then performed to prove the validity and correctness of the algorithm.

\section{SUBJECT \& METHODS}

\subsection{The establishment of multilateration measurement model}

A multilateration measurement method, based on a multistation laser tracker, effectively uses high-precision length measurement to precisely position the measuring point in space. In the actual measurement, test points are the ball center of the laser tracker's target mirror, so the laser tracker in the reference coordinate system can be viewed as a particle providing continuous stable laser output. As shown in Fig.1., distance between measuring point $T_{i}(x, y, z)$ and the particle $P_{k}(X, Y, Z)$ is interpreted as a spherical radius. So the laser displacement $l_{i k}$ varies always along the radial direction between the measuring point and the particle, regardless of the movement path of measurement point. When the measurement point from the initial measurement point $T_{0}(x, y, z)$ moves to any test point $T_{i}(x, y, z)$, the laser displacement is $l$, then according to two-point linear equation the spatial relationship can be established by the following equation [8]

$$
\sqrt{\left(x_{i}-X_{\mathrm{k}}\right)^{2}+\left(y_{i}-Y_{k}\right)^{2}+\left(z_{i}-Z_{k}\right)^{2}}=d_{k}+l_{i k}
$$

DOI: $10.1515 / \mathrm{msr}-2016-0025$ 


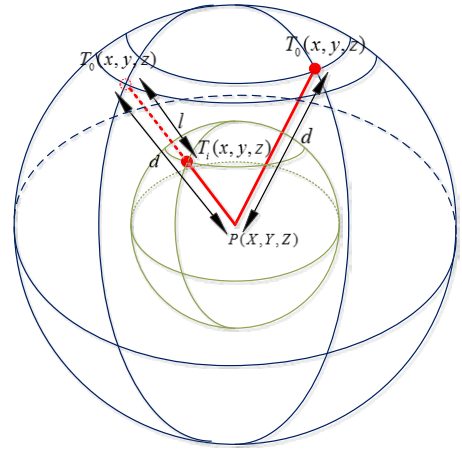

Fig.1. Laser tracker multilateration measurement model schematic ( $l$ is the laser displacement, $T_{0}(x, y, z)$ and $T_{i}(x, y, z)$ are the measurement points, $d$ is the dead path length, $P(X, Y, Z)$ is position coordinate of the laser trackers).

\subsection{Optimization model and solution}

The value of laser displacement $l_{i k}$ in (1) is usually regarded as the true value in laser tracker multilateration measurement methods; thus, from (1) the error equation can be written as

$$
v_{i k}=\left(\sqrt{\left(x_{i}-X_{\mathrm{k}}\right)^{2}+\left(y_{i}-Y_{k}\right)^{2}+\left(z_{i}-Z_{k}\right)^{2}}-d_{k}\right)-l_{i k}
$$

$\mathrm{V}_{\mathrm{ik}}$ is the residual between measured and fitted distance to measurement point.

Together with the position coordinates of the laser trackers $\left(X_{k}, Y_{k}, Z_{k}\right)$, they are the so-called system parameters, and collectively expressed as $P$. $\left(x_{i}, y_{i}, Z_{i}\right)$ are the coordinates of the measured targets, and collectively expressed as $T$. As an interferometer is a relative measurement device, measuring only changes in laser displacement $l_{i k .} . l_{i k}$ are the relative distance measurement of the $i$-th target point, as measured by the $k$-th laser tracker, and collectively expressed as $L$. A dead path length $d$ is introduced, collectively expressed as $D$, common to all distance measurements from the same laser tracker. In the usual solution model, the redundant equations are constituted, and then the least squares method is needed to solve the equations as follows.

$$
\begin{aligned}
& E(T, P, D)=\sum_{i=1} \sum_{k=1} v_{i k}^{2}(L, P, T, D)(i=1,2,3 \cdots n, \\
& k=1,2,3 \cdots m)
\end{aligned}
$$

There are many numerical algorithms for solving least squares problems, such as trust region method, GaussNewton method, Levenberg-Marquardt method, general inverse methods, and so on. However, an initial value is needed regardless of any numerical algorithm. In addition, the accuracy of the initial value selected may directly affect the calculation accuracy and efficiency. When the initial value is far from the true value, the iteration calculation may not converge, then the results cannot be obtained, so the selection of initial value is crucial. And the following method is used.

$\mathrm{n}$ is the number of the target points, $\mathrm{m}$ is the number of laser tracker stations. Therefore, the total of unknowns is
$3 n+4 m$, and each station can provide $n$ laser displacement values, a total of equations is $m \times n$. To ensure (2) can be solved, $m \times n \geq 3 n+4 m$ is a necessary limit. A linear expansion of (2) is as follows.

$$
\begin{aligned}
v_{i k}= & L_{i k}^{0}-d_{k}^{0}-l_{k}+\left.\frac{\delta v_{i k}}{\delta x_{i}}\right|_{0} \Delta x_{i}+\left.\frac{\delta v_{i k}}{\delta y_{i}}\right|_{0} \Delta y_{i} \\
& +\left.\frac{\delta v_{i k}}{\delta z_{i}}\right|_{0} \Delta z_{i}+\left.\frac{\delta v_{i k}}{\delta X_{i}}\right|_{0} \Delta X_{i} \\
& +\left.\frac{\delta v_{i k}}{\delta Y_{i}}\right|_{0} \Delta Y_{i}+\left.\frac{\delta v_{i k}}{\delta Z_{i}}\right|_{0} \Delta Z_{i}-\Delta d_{k}
\end{aligned}
$$

The simplified equation of (4) is as follows.

$$
\begin{aligned}
v_{i j}= & L_{i k}^{0}-d_{k}^{0}-l_{i k}+\frac{x_{i}^{0}-X_{k}^{0}}{L_{i k}^{0}} \cdot\left(\Delta x_{i}-\Delta X_{k}\right) \\
& +\frac{y_{i}^{0}-Y_{k}^{0}}{L_{i k}^{0}} \cdot\left(\Delta y_{i}-\Delta Y_{k}\right) \\
& +\frac{z_{i}^{0}-Z_{k}^{0}}{L_{i k}^{0}} \cdot\left(\Delta z_{i}-\Delta Z_{k}\right)-\Delta d_{k} \\
L_{i k}^{0}= & \left(\left(x_{i}^{0}-X_{k}^{0}\right)^{2}+\left(y_{i}^{0}-Y_{k}^{0}\right)^{2}+\left(z_{i}^{0}-Z_{k}^{0}\right)^{2}\right)^{1 / 2}
\end{aligned}
$$

Equation (5) is the optimization solution model. $\Delta x_{i}, \Delta y_{i}$, $\Delta z_{i}, \Delta X_{k}, \Delta Y_{k}, \Delta Z_{k}$ are coordinate correction values for target points and laser tracker station particle. $\Delta d_{k}$ is correction value of dead path length. Both of them are the unknowns. Those variables with superscripts of zero are initial values. So these variables may be the approximation, which can effectively reduce the precision influence of the selfcalibration. We can get the initial $x_{i}^{0}, y_{i}^{0}, z_{i}^{0}$ by reading the $3 \mathrm{D}$ coordinates of the measurement point in CMM. The initial value of the laser trackers' coordinates $X_{k}{ }^{0}, Y_{k}{ }^{0}, Z_{k}{ }^{0}$ and the dead path length $d_{k}{ }^{0}$ can be derived through the selfcalibration in the next section.

\subsection{Calibration of laser tracker station coordinate}

A measurement point coordinate might be temporarily regarded as the true value if only the approximate coordinates of a laser tracker station are needed as the expansion point to iterate. Thus, target point coordinate $T_{i}(x, y, z)$ and laser displacement $l_{i k}$ are the known values, and they are used to calibrate the coordinates $P_{k}(X, Y, Z)$ of the laser tracker at each station and the corresponding dead path length $d_{k . .}$ Taking the case of 1 st station as an example, (2) becomes

$$
\sqrt{\left(x_{i}-X_{1}\right)^{2}+\left(y_{i}-Y_{1}\right)^{2}+\left(z_{i}-Z_{1}\right)^{2}}=d_{1}+l_{i 1}
$$

In the same way, (7) written as the error is as follows.

$$
v_{i 1}=\left(\sqrt{\left(x_{i}-X_{1}\right)^{2}+\left(y_{i}-Y_{1}\right)^{2}+\left(z_{i}-Z_{1}\right)^{2}}-\left(d_{1}+l_{i 1}\right)\right.
$$


Suppose $a=X_{1}^{2}+Y_{1}^{2}+Z_{1}^{2}-d_{1}^{2}$, and use the least squares method to (8)

$$
\begin{aligned}
\frac{\partial v_{i 1}^{2}}{\partial X_{1}}= & \sum_{i=1}^{n} x_{i}\left(x_{i}^{2}+\mathrm{y}_{i}^{2}+\mathrm{z}_{i}^{2}-l_{i}^{2}\right)-2 \sum_{i=1}^{n} x_{i}^{2} X_{1}- \\
& 2 \sum_{i=1}^{n} y_{i} x_{i} Y_{1}-2 \sum_{i=1}^{n} z_{i} x_{i} Z_{1}-2 \sum_{i=1}^{n} l_{i} x_{i} d_{1}+\sum_{i=1}^{n} x_{i} a=0 \\
\frac{\partial v_{i 1}^{2}}{\partial Y_{1}}= & \sum_{i=1}^{n} \mathrm{y}_{i}\left(x_{i}^{2}+\mathrm{y}_{i}^{2}+\mathrm{z}_{i}^{2}-l_{i}^{2}\right)-2 \sum_{i=1}^{n} x_{i} y_{i} X_{1}- \\
& 2 \sum_{i=1}^{n} y_{i}^{2} Y_{1}-2 \sum_{i=1}^{n} z_{i} y_{i} Z_{1}-2 \sum_{i=1}^{n} l_{i} y_{i} d_{1}+\sum_{i=1}^{n} y_{i} a=0 \\
\frac{\partial v_{i 1}^{2}}{\partial Z_{1}}= & \sum_{i=1}^{n} z_{i}\left(x_{i}^{2}+\mathrm{y}_{i}^{2}+\mathrm{z}_{i}^{2}-l_{i}^{2}\right)-2 \sum_{i=1}^{n} x_{i} z_{i} X_{1}- \\
& 2 \sum_{i=1}^{n} y_{i} z_{i} Y_{1}-2 \sum_{i=1}^{n} \mathrm{z}_{i}^{2} Z_{1}-2 \sum_{i=1}^{n} l_{i} z_{i} d_{1}+\sum_{i=1}^{n} z_{i} a=0 \\
\frac{\partial v_{i 1}^{2}}{\partial d_{1}}= & \sum_{i=1}^{n} l_{i}\left(x_{i}^{2}+\mathrm{y}_{i}^{2}+\mathrm{z}_{i}^{2}-l_{i}^{2}\right)-2 \sum_{i=1}^{n} x_{i} l_{i} X_{1}- \\
& 2 \sum_{i=1}^{n} y_{i} l_{i} Y_{1}-2 \sum_{i=1}^{n} z_{i} l_{i} Z_{1}-2 \sum_{i=1}^{n} l_{i}^{2} d_{1}+\sum_{i=1}^{n} l_{i} a=0 \\
\frac{\partial v_{i 1}^{2}}{\partial a}=\sum_{i=1}^{n}\left(x_{i}^{2}+\mathrm{y}_{i}^{2}+\mathrm{z}_{i}^{2}-l_{i}^{2}\right)-2 \sum_{i=1}^{n} x_{i} X_{1}- & y_{i}-2 \sum_{i=1}^{n} z_{i} Z_{1}-2 \sum_{i=1}^{n} l_{i} d_{1}+\sum_{i=1}^{n} a=0 \\
&
\end{aligned}
$$

We can get the approximate coordinates of the laser tracker particle and the dead path length by simultaneous equations (9) to (13), and similarly for the other stations. All the values can be used as the initial expansion point in (5) to iterate. Substitute the approximate coordinates of the laser tracker particle at each station, measured coordinates of the target point, dead path length into (5), to get the coordinate correction values of each target point. Then add the coordinates of the target point and the correction values of each target point to acquire high-precision target point coordinate after correction.

\subsection{Singular value decomposition for solution model}

Simplify (5) into matrix equality as follows, after determining the initial value of each expansion point.

$$
A x=b
$$

where $x$ and $b$ are

$$
\begin{gathered}
x=\left[\Delta d_{1}, \Delta X_{1}, \Delta Y_{1}, \Delta Z_{1}, \cdots, \Delta d_{\mathrm{m}}, \Delta X_{m}, \Delta Y_{m}, \Delta Z_{m},\right. \\
\left.\cdots, \Delta x_{1}, \Delta y_{1}, \Delta z_{1}, \cdots, \Delta x_{n}, \Delta y_{n}, \Delta z_{n}\right]_{1 \times(3 n+4 m)}^{T}
\end{gathered}
$$

$$
\begin{aligned}
b= & {\left[l_{11}+d_{1}^{0}-L_{11}^{0}, l_{21}+d_{1}^{0}-L_{21}^{0}, \cdots, l_{n m}\right.} \\
& \left.+d_{m}^{0}-L_{n m}^{0}\right]_{1 \times n m}^{T}
\end{aligned}
$$

The matrix in (14) is a large sparse matrix, in which each row has seven non-zero parameters, and the remaining parameters are zero. Because the actual number of conditions is great during the solution, the usual Gaussian Elimination does not apply. So the matrix is needed for singular value decomposition. That is $A=U S V^{T}$, where $S$ is singular value diagonal matrix, $U \in S O(m \times n), \quad V \in S O$ $(3 n+4 m)$.

The singular value decomposition is used in (14), which seeks a vector to minimize $\|A x-b\|=\left\|U S V^{T} x-b\right\|$. $\left\|U S V^{T} x-b\right\|=\left\|S V^{T} x-U^{T} b\right\|$ is obtained by sign preserving properties of the orthogonal matrix, and $y=V^{T} x, b^{\prime}=U^{T} b$, so

$$
\left[\begin{array}{ccc}
s_{11} & 0 & 0 \\
0 & \ddots & 0 \\
0 & & s_{(3 n+4 m)(3 n+4 m)} \\
\vdots & & \vdots \\
0 & 0 & 0
\end{array}\right]\left(\begin{array}{c}
y_{1} \\
y_{2} \\
\vdots \\
\vdots \\
y_{3 n+4 m}
\end{array}\right)=\left(\begin{array}{c}
b_{1}^{\prime} \\
b_{2}^{\prime} \\
\vdots \\
\vdots \\
b_{m \times n}^{\prime}
\end{array}\right)
$$

From (17), we get $y_{i}=b_{i}^{\prime} / s_{i}(i=1,2, \cdots, 3 n+4 m)$.

According to $y=V^{T} x$, combined with the property of orthogonal matrix $x=V y$, corrections of measurement point coordinates $x$ can be obtained.

Actually, the singular value matrix $S$ is a characteristics reflection of matrix $A$. The external factors in the singular value matrix $S$ must be excluded in order to retain an important eigenvalue of coefficient matrix $A$ when environmental factors prominently influence the measurement results.

Introduce the parameter $\varepsilon$ reflecting the accuracy of the principal component of coefficient matrix $A$. And any element less than $\varepsilon$ in the singular value matrix $S$ can be ignored, which can reduce the condition number of the matrix, which better reflects the main element characteristic. The value $\varepsilon$ is determined by the following formula.

$$
\begin{gathered}
\varepsilon=(\text { the main element precision error threshold }) \\
\cdot \max \left\{s_{i}\right\}
\end{gathered}
$$

$S_{i}$ is the element of singular value matrix $S$, the main element precision error threshold, according to the actual measurement conditions, generally can be $10^{-2}$ to $10^{-4}$.

\section{RESULTS AND DISCUSSION}

\subsection{Experimental verification}

We have conducted the following experiment in order to verify the effectiveness of the measurement model. 



Fig.2. Experimental arrangement.

Fig.2. delineates the experiment prototype. A laser tracker, LaserTRACER by Etalon, measured 36 target points provided by a CMM successively at 5 stations [10]-[14]. Since only one laser tracker was used, the same measurement was repeated with the laser tracker set at five different positions on the table, assuming that the CMM's unrepeatable positioning error was sufficiently small. The motion region of target points was defined by $600 \mathrm{~mm} \times 300 \mathrm{~mm} \times 300 \mathrm{~mm}$, and the No.1 point in the $1 \mathrm{st}$ floor was the coordinates of $(600.498 \mathrm{~mm}, 550.831 \mathrm{~mm}$,$550.738 \mathrm{~mm}$ ). Total measurement points were three planes with 12 target points in each plane. Meanwhile, 12 measuring points were uniformly distributed in each edge of the square. In the measurement process, the target point moved along the preset path, while laser tracker tracked its movement real-time. When the target point reached each measuring point, it stopped and the distance reading of laser tracker was written down at the corresponding position.

Each of the coordinate corrections obtained by laser tracker multilateration optimization solution model was plotted as shown in Fig.3. As revealed in the figure, threeaxis correction fluctuation trend did not change significantly, which was consistent with the normal error characteristics of the CMM. The experiment obtained corrections for the target point coordinates, and demonstrated the optimization solution model's capability of precision correction. However, the experiment did not prove correctness of the described solution model. Thus, the following supplementary experiment has been performed based on the above.

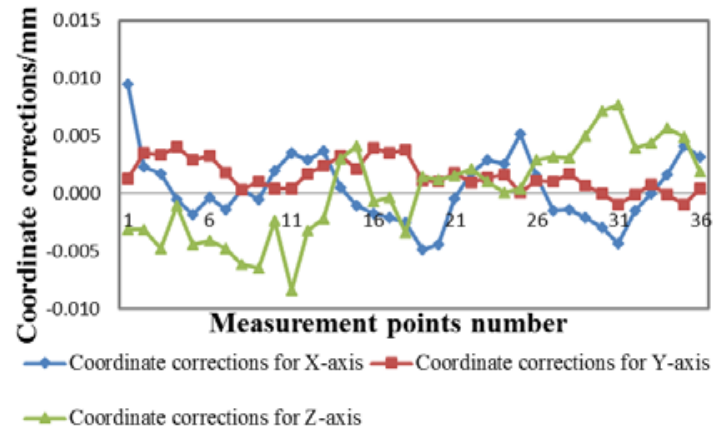

Fig.3. The corrections of point coordinate obtained by multilateration optimization solution model.

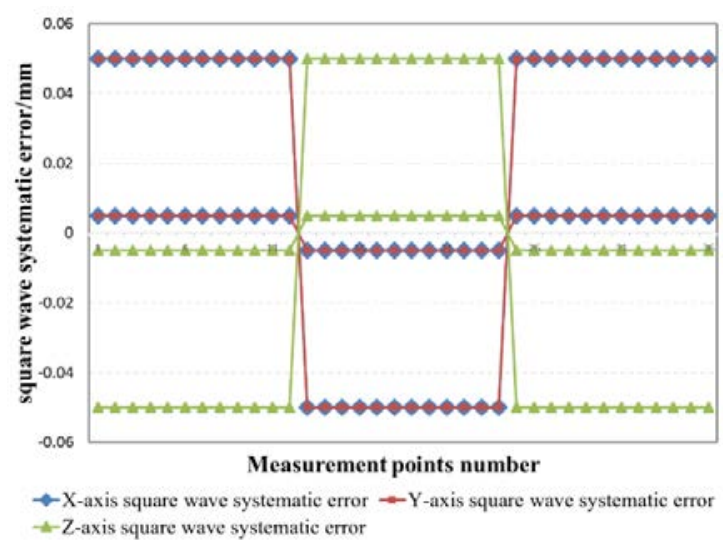

Fig.4. Square wave systematic error.

We added an error to the CMM target points. The normal measurement error sources included random and systematic errors, and the former was not easily observable. The square wave system error, $0.005 \mathrm{~mm}$ and $0.05 \mathrm{~mm}$, respectively, had been introduced to illustrate the validity of the optimization model, as denoted in Fig.4.

After introducing the $0.005 \mathrm{~mm}$ and $0.05 \mathrm{~mm}$ square wave system error, respectively, we applied the multilateration measurement optimization model. These coordinate corrections are shown in Fig.5. and Fig.6.

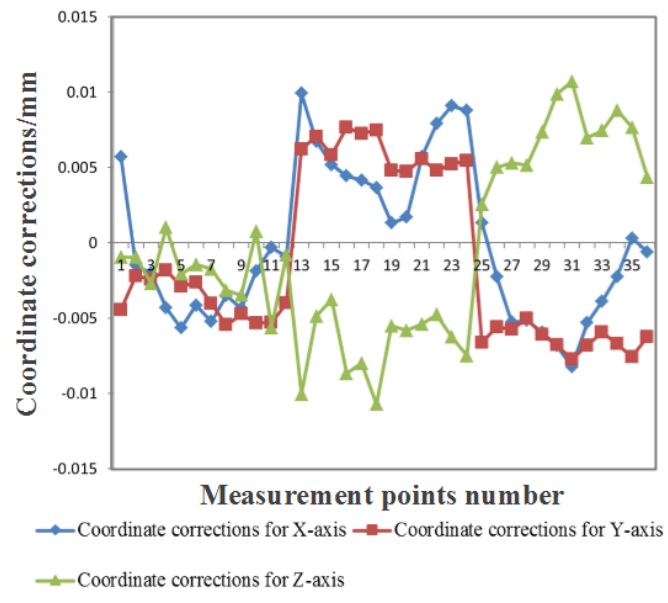

Fig.5. Coordinate corrections after introduction of $0.005 \mathrm{~mm}$ error. 




Fig.6. Coordinate corrections after introduction of $0.05 \mathrm{~mm}$ error.

As illustrated in Fig.5. and Fig.6., corrections for each axis were significantly greater than without introducing a square wave system error. The maximum fluctuation range of corrections was roughly $-0.008 \mathrm{~mm} \sim+0.008 \mathrm{~mm}$ before introducing the error, and with $0.005 \mathrm{~mm}$ square error it became $-0.01 \mathrm{~mm} \sim+0.01 \mathrm{~mm}$, and with $0.05 \mathrm{~mm}$ square error the range correction was $-0.06 \mathrm{~mm} \sim+0.06 \mathrm{~mm}$. The trend of corrections should offset error trend in Fig.4., namely the corrections for $X$-axis and $Y$-axis of points in the 1st plane have to be negative, $Z$-axis should be positive. Similarly, for points in the 2nd plane, the corrections for $X$ axis and $Y$-axis coordinate ought to be positive, $Z$-axis should be negative. And for points in the 3rd plane, the corrections for $X$-axis and $Y$-axis coordinate ought to be negative, Z-axis should be positive. Fig.5. and Fig.6. illustrate distinctly that coordinate corrections meet the above trends.

Notably in Fig.5., the trend of corrections for points in the 1st plane was disordered, and the overall trend of corrections was not as clear as the trend in Fig.6. The reason for this phenomenon was that the accuracy range of CMM had been beyond $0.005 \mathrm{~mm}$ before introducing the square system error. And the corrections fluctuate from $-0.008 \mathrm{~mm}$ to $+0.008 \mathrm{~mm}$. If signal intensity of the systematic error and the original random error was about the same, the superposition of them made trend curve of corrections to be as shown in Fig.5. After the introduction of $0.05 \mathrm{~mm}$ square wave systematic error, which was greater than the original random error, the square error dominated. Therefore, the curve of error correction in Fig.6. was superior to that in Fig.5., which was in line with the actual situation.

A comparison experiment between the method proposed and the method proposed in reference [8] was also done to further validate the model. The result is shown in Fig.7. Experiment shows that two results coincide well. Thus, error corrections acquired by the multilateration measurement optimization solution model were of correctness and validity.

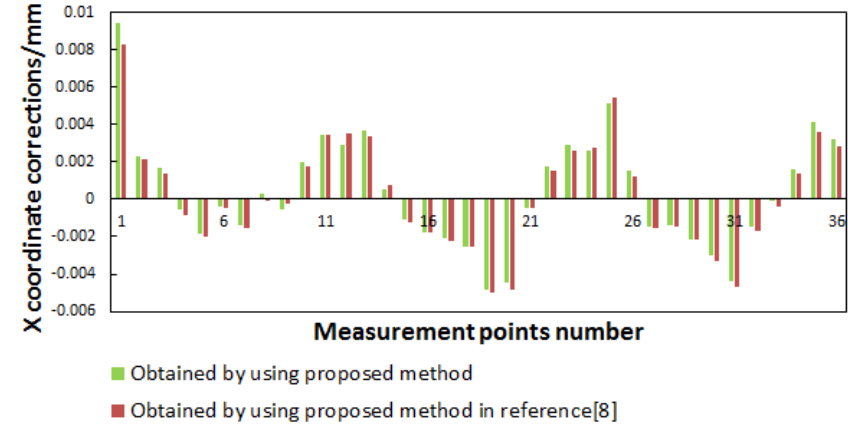

Fig.7. The comparison between two methods.

\section{CONCLUSIONS}

The optimization solution model for a laser tracker multilateration measurement, that effectively inhibits the negative effect of accuracy induced by self-calibration, is described. The accuracy of singular value decomposition for the solution model is further analyzed. We performed verification experiment of the solution model based on laser tracker and CMM, using the coordinate error of measurement points in the CMM as evaluation criteria, and compared the results of multilateration measurement with and without the introduction of artificial system error. The experiment results show that the optimization solution model of laser tracker multilateration measurement described in this paper can effectively enhance accuracy, which has potentially broad applicability in the field of laser tracker spatial orientation.

\section{ACKNOWLEDGMENT}

The research is supported by National Key Scientific Instrument and Equipment Development Project of China (No.2013YQ17053904), National Science and Technology Major Project of China (No. 2010ZX04014-091) and National Natural Science Foundation of China ( No.50805003 ).

\section{REFERENCES}

[1] Sharke, P. (2003). Measuring across space and time: Large-scale metrology moves GPS in out of the rain. Mechanical Engineering, 125 (1), 48.

[2] Lin, J., Zhu, J., Guo, Y., Guo, T., Ye, S. (2012). Establishment of precise three-dimensional coordinate control network in field large-space. Chinese Journal of Mechanical Engineering, 48 (4), 6-11.

[3] Hughes, B., Forbes, A., Lewis, A., Sun, W., Veal, D., Nasr, K. (2011). Laser tracker error determination using a network measurement. Measurement Science and Technology, 22, 1-12.

[4] Hughes, E.B., Wilson, A., Peggs, G.N. (2000). Design of a high-accuracy CMM based on multilateration techniques. CIRP Annals - Manufacturing Technology, 49 (1), 391-394. 
[5] Takatsuji, T., Goto, M., Kirita, A. (2000). The relationship between the measurement error and the arrangement of laser trackers in laser trilateration. Measurement Science and Technology, 11 (5), 477483.

[6] Zhang, D., Rolt, S., Maropoulos, P.G. (2005). Modeling and optimization of novel laser multilateration schemes for high-precision applications. Measurement Science and Technology, 16, 2541-2547.

[7] Wang, J., Guo, J. (2011). Method of geometric error identification for numerical control machine tool based on laser tracker. Chinese Journal of Mechanical Engineering, 14, 13-19.

[8] Wang, J., Guo, J. (2013). Algorithm for detecting volumetric geometric accuracy of NC machine tool by laser tracker. Chinese Journal of Mechanical Engineering, 26, 166-175.

[9] Wang, J., Guo, J., Zhang, G. (2012). The technical method of geometric error measurement for multi-axis NC machine tool by laser tracker. Measurement Science and Technology, 23, 1-11.

[10] Umetsu, K., Furutani, R., Takatsuji, T., Osawa, S., Kurosawa, T. (2003). Calibration of a CMM using a laser tracking system. In XVII IMEKO World Congress, June 22-27, 2003, Dubrovnik, Croatia. TC14, 1860-1863.
[11] Muralikrishnan, B., Sawyer, D., Blackburn, C., Phillips, S. (2009). ASME B89.4.19 performance evaluation tests and geometric misalignments in laser trackers. Journal of Research of the National Institute of Standards and Technology, 114, 21-35.

[12] Schwenke, H., Schmitt, R., Jatzkowski, P., Warmann, C. (2009). On-the-fly calibration of linear and rotary axes of machine tools and CMMs using a tracking interferometer. CIRP Annals - Manufacturing Technology, 58, 477-480.

[13] Schwenke, H., Frank, M., Hannaford, J. (2005). Error mapping of CMMs and machine tools by a single tracking interferometer. CIRP Annals - Manufacturing Technology, 54 (1), 475-478.

[14] Härtig, F., Keck, C., Kniel, K., Schwenke, H. (2003). Improvement of measurement accuracy by combined evaluation of CMM and tracking interferometer measurement. In XVII IMEKO World Congress, June 22-27, 2003, Dubrovnik, Croatia. TC7, 1172-1175.

Received January 03, 2016. Accepted August 10, 2016. 